\title{
CRP and the Prognosis of Patients with Cirrhosis
}

\section{Nicoleta STATE}

"Sf. Ioan cel Nou" Emergency Hospital of Suceava, Suceava, Romania

"Carol Davila" University of Medicine and Pharmacy, Bucharest, Romania

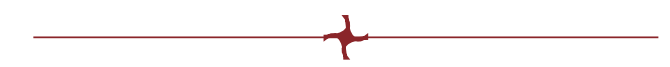

\begin{abstract}
-ABSTRACT
The theory of vasodilation partially explains the development and progression of liver cirrhosis and is completed by the theory of inflammation. C-reactive protein $(C R P)$ is used as a surrogate marker of inflammation in studies; however, there is not sufficient data that would reflect the role of this protein in cirrhosis yet.

The objective of this study was to determine the use of CRP value in the prognosis of patients with cirrhosis.

In "Material and method" section we described a clinical prospective trial with 102 participants represented by patients with cirrhosis of various etiologies in a tertiary hospital, each monitored throughout a period of 12 months after the collection of CRP.

The results showed that the mean CRP value was $0.7+/ 0.09 \mathrm{mg} / \mathrm{dL}$ (CI 0.59-0.90) in patients who did not decompensate throughout the trial and 1.58+/0.4 mg/dL (CI 1.76-2.30) in those who did decompensate, with a statistically significant difference ( $p=0.045$ ).

In rehospitalized patients versus those without any hospitalization, the mean CRP values were $1.35 \mathrm{mg} / \mathrm{dL}$ and $0.8 \mathrm{mg} / \mathrm{dL}$, respectively $(\mathrm{p}=0.032)$. The increased values of this parameter were positively correlated with the number of hospitalizations ( $r s=0.35, p=0.05$ ).

A CRP value below the threshold of $0.62 \mathrm{mg} / \mathrm{dL}$ indicates a smaller probability of future decompensation in liver cirrhosis patients.

The conclusion of this study is that CRP influences the secondary endpoints, including cirrhosis decompensation and patient rehospitalization. It can be added to the existing studies.
\end{abstract}

Keywords: CRP, cirrhosis, prognosis, decompensation, rehospitalization.

\section{INTRODUCTION}

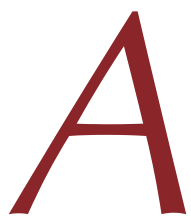
$s$ inflammation marker, the C-reactive protein (CRP) is used in practice to monitor several conditions: autoimmune diseases, cancer, acute infectious diseases. The usefulness of the CRP is maintained in cirrhosis as well. Despite the liver function deterioration, C-reactive protein synthesis is maintained in advanced stages (1). C-reactive protein is considered a surrogate marker of acute or chronic systemic inflammation and bacterial infections. Systemic inflammatory response syndrome (SIRS), with or without the documentation of a bacterial infection, is an independent factor for survival and is associated

Address for correspondence:

Nicoleta State

14 Putna Str., Bl A01, Sc B, Ap. 7, Suceava, Romania

Email:dr.state.nicoleta@gmail.com

Article received on the $9^{\text {th }}$ of April 2021 and accepted for publication on the 10 $0^{\text {th }}$ of August 2021

Maedica $\mid$ A Journal of Clinical Medicine, Volume 16, No. 3, 2021353 
with complications of portal hypertension (PHT) (2). In cirrhosis, the inflammation theory explains aspects which have not been clarified by the pathogenesis demonstrated through the vasodilation theory.

Intestinal dysbiosis (a disturbance of the saprophytic and pathogenic bacteria balance) was noticed in patients with cirrhosis by an increase of the microflora, and translocation of bacteria and derived bacterial products through the intestinal barrier. It is believed this process leads to systemic inflammation and dysfunction of the immune system. Consequently, it makes the patient susceptible to infections and complications of cirrhosis. Moreover, dysbiosis is believed to influence the progression of hepatic diseases $(3,4)$.

Currently, there are several prognosis factors for liver cirrhosis which constitutes the elements of known scores, the most important being Child Pugh and MELD (model of end stage liver disease) with its variants. Although they have proved their usefulness in time and considerable scientific evidence has been gathered, these scores present certain limits. The first one has the disadvantage of subjectively interpreting some variables. The other one is using only objective elements but despite this, it is still ascertained that $15-20 \%$ of cases remain erroneously classified (5); also, MELD is not used to assess patients' universal prognosis but to prioritize patients for hepatic transplant (6-8).

The objective of this study was to determine the role of CRP value in the prognosis of patients with liver cirrhosis.

\section{MATERIAL AND METHOD}

$\mathrm{B}$ ased on inclusion and exclusion criteria, 102 patients with known cirrhosis from the medical departments of a tertiary hospital were included in the trial. In addition, patients with newly diagnosed liver cirrhosis were enrolled in the trial.

Patients with neoplasia, those with severe hepatic encephalopathy, which impedes understanding and signing the informed consent, as well as those with a general altered state were excluded from the trial upon enrollment.

The patient group was heterogeneous with respect to cirrhosis etiology and subjects' age and stages of the disease. Some patients had de- compensated cirrhosis (considered when variceal hemorrhage, encephalopathy, icterus or ascites were present) (9), and the remaining ones compensated cirrhosis.

In patients presenting clinical signs of infections, blood culture was performed to exclude

TABLE 1. Characteristics of patients included in the study. BMI=body mass index, $\mathrm{SBP}=$ systolic blood pressure, $\mathrm{DBP}=$ diastolic blood pressure, $H R=$ heart rate, $L H L=l e f t$ hepatic lobe, RHL=right hepatic lobe, $P V D=$ portal vein diameter, IVC=inferior vena cava diameter, $\mathrm{SV}=$ splenic vein diameter, $\mathrm{T}=$-total bilirubin, INR=international normalized ratio, $\mathrm{Na}=$ sodium, $\mathrm{Hb}=$ haemoglobin, $\mathrm{Tr}=$ thrombocytes, Le=leucocytes, $\mathrm{MCV}=$ mean corpuscular volume, $\mathrm{AST}=$ aspartate aminotransferase, $\mathrm{ALT}=$ alanine aminotransferase, GGT=gamma glutamyl transferase, $\mathrm{AFP}=\alpha$-fetoprotein)

\begin{tabular}{|l|l|}
\hline Characteristic & Mean and standard deviation \\
\hline Age & $63.59 \pm 11.58$ \\
\hline Weight $(\mathrm{kg})$ & $82.28 \pm 19.36$ \\
\hline Height $(\mathrm{cm})$ & $165.50 \pm 10.99$ \\
\hline BMI & $30.02 \pm 10.99$ \\
\hline Dg from CH (years) & $3.61 \pm 5.73$ \\
\hline SBP $(\mathrm{mm} \mathrm{Hg})$ & $124.36 \pm 16.95$ \\
\hline DBP $(\mathrm{mm} \mathrm{Hg})$ & $67.74 \pm 10.91$ \\
\hline HR $($ bpm) & $80.25 \pm 13.73$ \\
\hline LHL $(\mathrm{mm})$ & $82.03 \pm 16.34$ \\
\hline RHL $(\mathrm{mm})$ & $168.91 \pm 21.08$ \\
\hline PVD $(\mathrm{mm})$ & $14.13 \pm 1.84$ \\
\hline IVC $(\mathrm{mm})$ & $21.16 \pm 5.29$ \\
\hline Spleen $(\mathrm{mm})$ & $143.16 \pm 23.34$ \\
\hline SV $(\mathrm{mm})$ & $7.94 \pm 2.40$ \\
\hline CRP $(\mathrm{mg} / \mathrm{dL})$ & $1.18 \pm 2.05$ \\
\hline Creatinine $(\mathrm{mg} / \mathrm{dL})$ & $1.09 \pm 0.65$ \\
\hline TB $(\mathrm{mg} / \mathrm{dL})$ & $2.03 \pm 2.53$ \\
\hline INR & $1.59 \pm 0.51$ \\
\hline Na $(\mathrm{mmol} / \mathrm{L})$ & $137.67 \pm 4.44$ \\
\hline MELD & $16.27 \pm 5.73$ \\
\hline Hb $(\mathrm{g} / \mathrm{dL})$ & $11.08 \pm 2.76$ \\
\hline Tr $($ thousands/ $\mu \mathrm{L})$ & $170.21 \pm 83.65$ \\
\hline Le (thousands/ $\mu \mathrm{L})$ & $6.25 \pm 3.03$ \\
\hline MCV $(\mathrm{fL})$ & $92.21 \pm 11.97$ \\
\hline Total cholesterol $(\mathrm{mg} / \mathrm{dL})$ & $134.89 \pm 44.15$ \\
\hline AST $(\mathrm{U} / \mathrm{L})$ & $79.17 \pm 174.75$ \\
\hline ALT $(\mathrm{U} / \mathrm{L})$ & $56.39 \pm 216.94$ \\
\hline Glycaemia $(\mathrm{mg} / \mathrm{dL})$ & $125.49 \pm 53.73$ \\
\hline GGT $(\mathrm{U} / \mathrm{L})$ & $167.62 \pm 208.60$ \\
\hline Total serum protein $(\mathrm{g} / \mathrm{dL})$ & $6.89 \pm 0.93$ \\
\hline Albumin $\%$ & $48.91 \pm 6.96$ \\
\hline AFP $(\mathrm{ng} / \mathrm{mL})$ & $5.30 \pm 9.31$ \\
\hline Serum iron $(\mu \mathrm{g} / \mathrm{dL})$ & $79.66 \pm 49.10$ \\
\hline$\%$ Prothrombin & $60.52 \pm 17.63$ \\
\hline Quick time $(\mathrm{sec})$ & $18.28 \pm 5.06$ \\
\hline Serum uric acid $(\mathrm{mg} / \mathrm{dL})$ & $6,70 \pm 2.03$ \\
\hline & \\
\hline
\end{tabular}




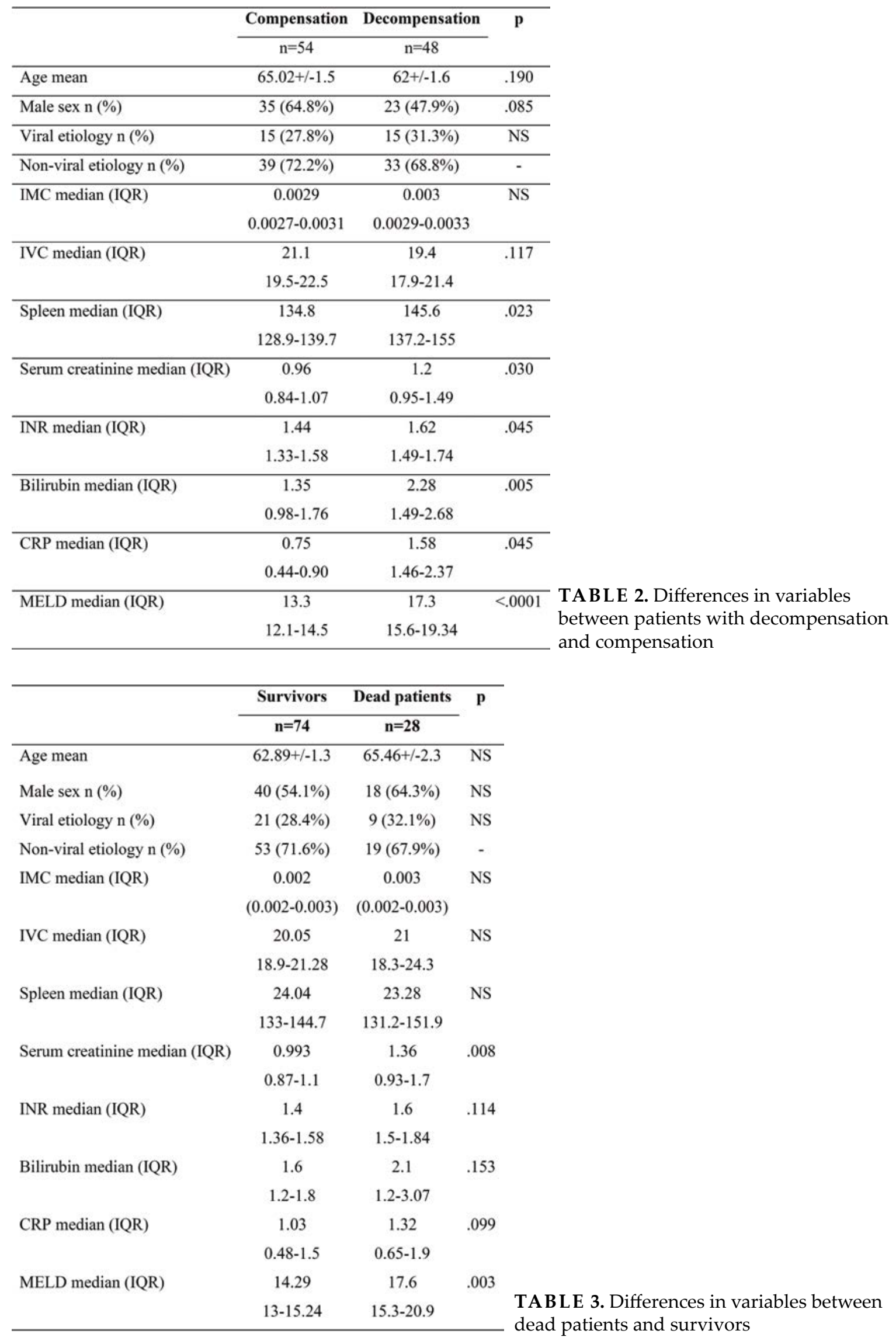




\begin{tabular}{|c|c|c|c|}
\hline & $\begin{array}{c}\text { Rehospitalized } \\
\text { patients }\end{array}$ & $\begin{array}{c}\text { Non-hospitalized } \\
\text { patients }\end{array}$ & p \\
\hline & $\mathrm{n}=63$ & $n=39$ & \\
\hline Age mean & $64.78+/-1.46$ & $61.69+/-1.8$ & 0.192 \\
\hline Male sex n (\%) & $32(50.8 \%)$ & $26(66.7 \%)$ & 0.116 \\
\hline Viral etiology n (\%) & $24(38.1 \%)$ & $6(15.4 \%)$ & 0.014 \\
\hline Non-viral etiology n (\%) & $39(84.6 \%)$ & $33(61.9 \%)$ & - \\
\hline \multirow[t]{2}{*}{ IMC median (IQR) } & 0.0029 & 0.003 & NS \\
\hline & $0.0020-0.0031$ & $0.0027-0.0032$ & \\
\hline \multirow[t]{2}{*}{ IVC median (IQR) } & 20.1 & 20.5 & NS \\
\hline & $18.6-21.7$ & $19-22.1$ & \\
\hline \multirow[t]{2}{*}{ Spleen median (IQR) } & 134 & 143.1 & 0.107 \\
\hline & $128.2-139.7$ & $135.8-150.5$ & \\
\hline \multirow[t]{2}{*}{ Serum creatinine median (IQR) } & 0.87 & 1.2 & 0.006 \\
\hline & $0.78-0.97$ & $1-1.41$ & \\
\hline \multirow[t]{2}{*}{ INR median (IQR) } & 1.4 & 1.5 & NS \\
\hline & $1.32-1.66$ & $1.5-1.65$ & \\
\hline \multirow[t]{2}{*}{ Bilirubin median (IQR) } & 1.2 & 1.9 & 0.009 \\
\hline & $1-1.46$ & $1.45-2.53$ & \\
\hline \multirow[t]{2}{*}{ CRP median (IQR) } & 0.57 & 1.4 & .032 \\
\hline & $0.44-0.69$ & $0.75-2.17$ & \\
\hline \multirow[t]{2}{*}{ MELD median (IQR) } & 13 & 16.5 & .003 \\
\hline & $11.5-14.4$ & $15-18$ & \\
\hline
\end{tabular}

TABLE 4. Differences in variables between rehospitalized and non-hospitalized patients

infections. However, the necessity of ruling out infections is no longer mandatory since it was proven that, regardless of the inflammation cause in cirrhosis patients, it does not change their evolution (10).

Once the patient consents obtained, CRP and other laboratory parameters were analyzed (Table 1). Patients were assessed by means of transabdominal ultrasound. All data from previous observation charts and medical documents found in the hospital archive or patient's personal archive were obtained.

This study was carried out with the approval of "Sf. Ioan cel Nou" Suceava Hospital Ethics Committee, approval registration number 9232/2017.

Given that we carried out an observational study, participants did not receive any therapeutic interventions. They were monitored for 12 months, noting complications and deaths. Subjects were invited to come for follow-up at one month and three, six and twelve months after the beginning of the study. Clinical, biological and radiological (transabdominal ultrasound) data were collected during each follow-up visit (Tables 1, 2, 3 and 4).

Microsoft Excel and the IBM SPSS Statistics 20 software were used for the statistical analysis.

To corroborate the observed results, a confirmatory analysis was carried out by estimating the concordance (c)-statistic (the area under receiver operating characteristic curve) of the MELD score and models adding CRP to the MELD score for predicting the primary endpoint (death) and secondary endpoint, including decompensation and rehospitalization. This also allowed us to identify the best score cut point, which maximized the sensitivity, specificity, and predictive values. A c-statistic between 0.8 and 0.9 indicates an excellent diagnostic accuracy, given that a c-statistic greater than 0.7 is generally considered a useful test. Quantitative variables were expressed by mean (SD) or median and qualitatively variables by percentage. To compare continuous variables, we used t-tests. The cutoff CRP values were analyzed by receiver operating characteristics curve, and the best accuracy of cutoff values was chosen. The Pearson correlation test was used to determine the correlation between 
the time to the first decompensation episode and CRP as well as the number of hospitalized episodes and CRP.

\section{RESULTS}

Datients' general characteristics, the mean and standard deviations of the clinical, laboratory, and abdominal ultrasound parameters upon enrollment were included in Tables 1, 2, 3 and 4.

Mortality, as primary endpoint, was not influenced by CRP (normal values $<0,5 \mathrm{mg} / \mathrm{dL}$ ). The CRP mean values were not associated with an increase in mortality $(1.03 \mathrm{mg} / \mathrm{dL}$ in those who survived versus $1.3 \mathrm{mg} / \mathrm{dL}$ in those who died, $p=0.09$ ). However, this proved to be useful regarding the secondary endpoints, including decompensation and rehospitalization.

The mean CRP value was greater among patients with decompensation compared to those who did not decompensate. Patients who did not decompensate throughout the trial had a mean CRP value of $0.75 \mathrm{mg} / \mathrm{dL}(\mathrm{Cl} 0.44-0.9)$. Patients with decompensation had a mean CRP value of $1.58 \mathrm{mg} / \mathrm{dL}(\mathrm{Cl} 1.46-2.3)$, a statistically significant difference $(p=0.045)$ (Figure 1).

Logistic regression analysis to investigate the predictive value of CRP and MELD on the secondary endpoints was conducted. The predictor variable, CRP and MELD were tested aprioristic to verify that there was no violation of assumption of the logit linearity. The beta weight for the MELD only was insignificant $(B=0.14$,

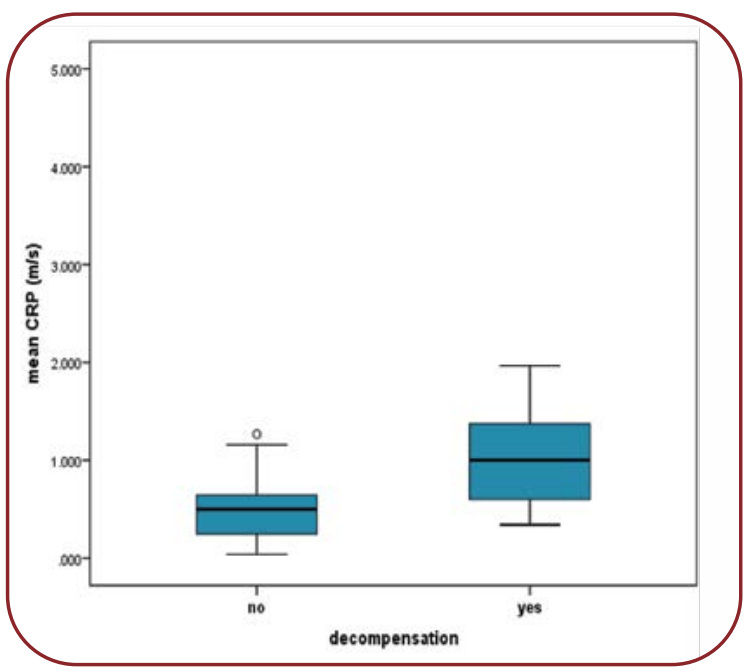

FIGURE 1. Mean CRP differences between decompensation and compensation
Wald $=5.89, p=0.015)$ and estimated odds ratio $\operatorname{Exp}(\mathrm{B}) 1.15,95 \% \mathrm{Cl}$ (1.02-1.28). CRP added to the model increases the estimated odds ratio to 3.36 for $95 \% \mathrm{Cl}(1.23-8.75)$ (Table 5).

MELD*CRP $=0.14$ (MELD)+1.2(CRP)-3.16.

Using CRP together with MELD, the c-statistic improved from $0.71(<.0001)$ to $0.79(<.0001)$. For CRP only a cut-off value of 0.75 was identified, which corresponded to a sensitivity of $73.8 \%$ and a specificity of $58 \%$ (Figure 2 ).

TABLE 5. Predicted values for decompensation of CRP and MELD

\begin{tabular}{|c|c|c|c|c|c|c|c|c|c|}
\hline & & & & & & & & $95 \%$ CI & for $\operatorname{Exp}(B)$ \\
\hline & & B & S. E & Wald & df & Sig. & $\operatorname{Exp}(B)$ & Lower & Upper \\
\hline Step 1 ${ }^{\text {a }}$ & MELD & .14 & .06 & 5.89 & 1 & .015 & 1.15 & 1.02 & 1.28 \\
\hline Step $2^{b}$ & MELD*CRP & 1.20 & .49 & 5.96 & 1 & .045 & 3.36 & 1.15 & 7.75 \\
\hline
\end{tabular}

a. Variable(s) entered on step 1: MELD, CRP

b. Variable(s) entered on step 2: MELD, CRP

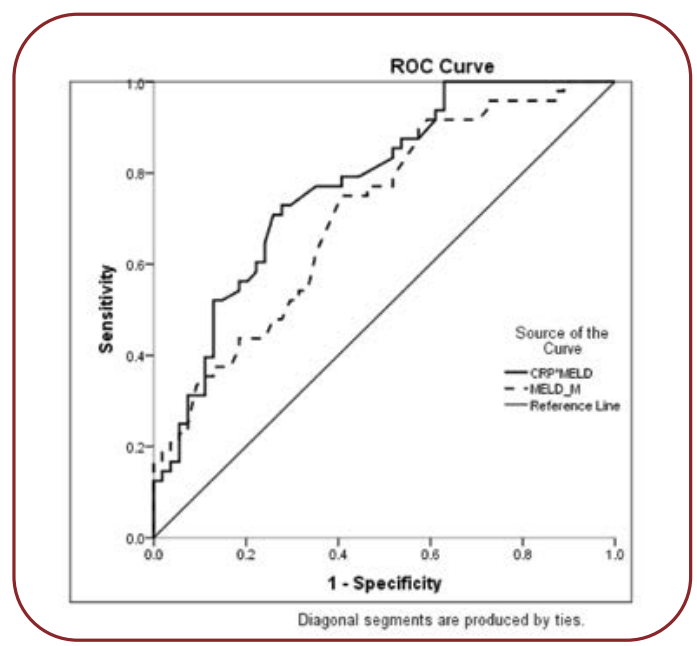

FIGURE 2. Predictive value of MELD and MELD*CRP for decompensation

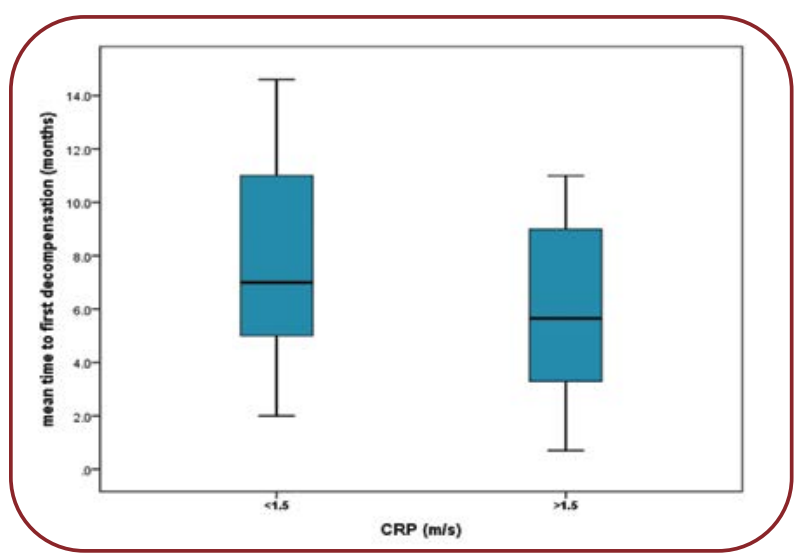

FIGURE 3. Mean time differences to first decompensation episode for two categories of CRP 
The mean duration up to the first decompensation is lower among patients with an average CRP of $>1.5 \mathrm{mg} / \mathrm{dL}$ versus CRP $<1.5 \mathrm{mg} / \mathrm{dL}(6.7$ months versus 7.2 months, $p=0.07$ ) (Figure 3 ).

The elevated CRP level was positively correlated with an increased number of decompensations ( $r s=0.45, p=0.044$ ).

An increase in the predictive accuracy to $65 \%$ was seen by adding CRP to the MELD score,

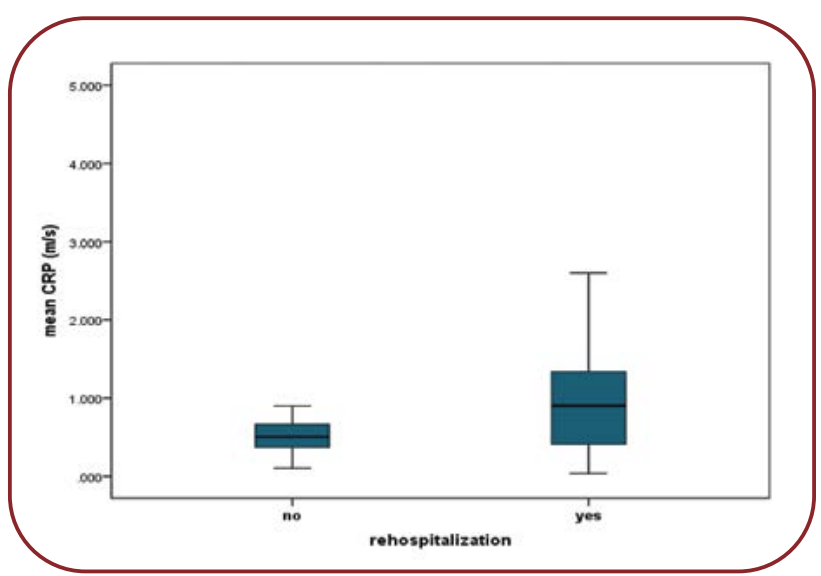

FIGURE 4. Mean CRP differences between rehospitalized and non-hospitalized

TABLE 6. Predicted value for decompensation of CRP and MELD

95\% CI for $\operatorname{Exp}(\mathrm{B})$

\begin{tabular}{lllllllllll} 
& \multicolumn{1}{c}{ B } & \multicolumn{2}{c}{ S.E. Wald df Sig. } & $\operatorname{Exp}(\mathbf{B})$ & Lower & Upper \\
\hline Step 1 $^{\text {a }}$ & MELD & .115 & .056 & 4.125 & 1 & .042 & 1.12 & 1.004 & 1.252 \\
Step 2" & CRP & 1.319 & .534 & 4.552 & 1 & .050 & 2.03 & 1.301 & 4.473
\end{tabular}

a. Variable(s) entered on step 1: MELD

b. Variable(s) entered on step 2: CRP

TABLE 7. Likelihood ratio tests

\begin{tabular}{lcccc}
\hline & \multicolumn{2}{c}{ Model fitting criteria } & \multicolumn{3}{c}{ Likelihood ratio tests } \\
\cline { 2 - 6 } Effect & $\mathbf{- 2}$ Log likelihood of reduced model & Chi-square & df & Sig. \\
\hline Intercept & $14.881^{\mathrm{a}}$ & .000 & 0 &. \\
MELD_categ & 21.269 & 6.388 & 1 & .011 \\
CRP categ & 22.720 & 7.839 & 1 & .005 \\
\hline \hline
\end{tabular}

TAB LE 8. Parameter estimates

\begin{tabular}{|c|c|c|c|c|c|c|c|}
\hline \multirow[b]{2}{*}{ Decompensation ${ }^{a}$} & \multirow[b]{2}{*}{ B } & \multirow[b]{2}{*}{ Std. error } & \multirow[b]{2}{*}{ Wald } & \multirow[b]{2}{*}{ df } & \multirow[b]{2}{*}{$\operatorname{Exp}(B)$} & \multicolumn{2}{|c|}{ 95\% CI for $\operatorname{Exp}(\mathrm{B})$} \\
\hline & & & & & & Lower bound & Upper bound \\
\hline nu Intercept & -.894 & .396 & 5.103 & 1.024 & & & \\
\hline $\begin{array}{l}{[\text { MELD_categ }=0]} \\
{[\text { CRP_categ }=0]}\end{array}$ & 2.434 & .599 & $\begin{array}{r}16.50 \\
8\end{array}$ & 1.000 & 11.407 & 3.525 & 36.911 \\
\hline $\begin{array}{l}{[\text { MELD_categ }=0]} \\
{[\text { CRP_categ }=1]}\end{array}$ & .488 & .757 & .416 & 1.519 & 1.630 & .370 & 7.187 \\
\hline $\begin{array}{l}{[\text { MELD_categ }=1]^{*}} \\
{[\text { CRP_categ }=0]}\end{array}$ & .894 & .557 & 2.574 & 1.109 & 2.444 & .820 & 7.285 \\
\hline $\begin{array}{l}{[\text { MELD_categ }=1] *} \\
{[\text { CRP_categ }=1]}\end{array}$ & $0^{b}$ & • & & 0 & . & . & . \\
\hline
\end{tabular}

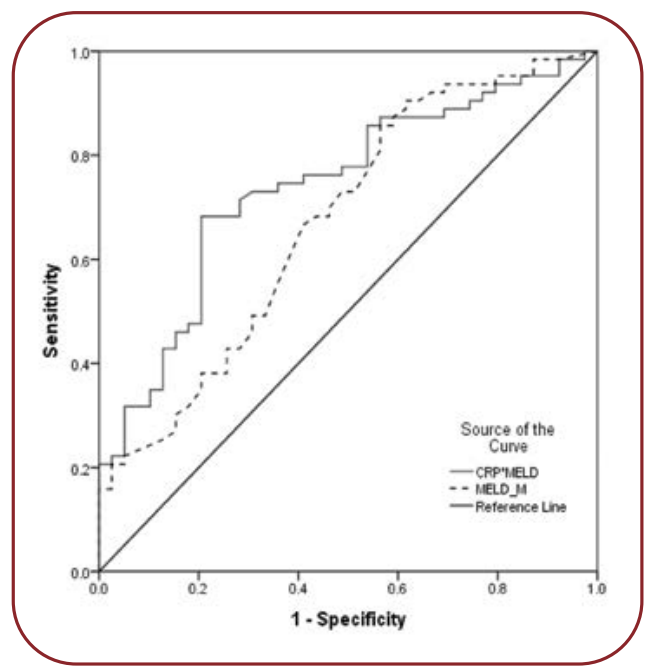

FIGURE 5. Mean CRP differences between rehospitalized and non-hospitalized

with a sensitivity of $63 \%$ and a specificity $65 \%$, $\mathrm{OR}=1.37(\mathrm{Cl} 1.09-1.2, \mathrm{p}=0.04)$.

Regarding hospitalization, average CRP in subjects with repeated hospitalizations registered slightly increased average values, of $1.35 \mathrm{mg} / \mathrm{dL}$, compared to patients without rehospitalizations, where the mean value was of $0.8 \mathrm{mg} / \mathrm{dL}$ $(p=0.032)$. The increased values of this parameter were positively correlated with the number of hospitalizations ( $r s=0.35, p=0.05)$. The predictive value of CRP was reduced, with an accuracy of just $60 \%$ and a relative risk of $\mathrm{OR}=2.03$ (Cl 1.3-4.4, $\mathrm{p}=0.05)$ (Figure 4).

By addition to MELD score, c-statistic improved from $0.453(p=0.042)$ to 0.672 $(p=0.033)$ (Table 6, Figure 5).

$$
\text { MELD*CRP }=0.115(\mathrm{MELD})+1.139(\mathrm{CRP})-
$$
2.04

Adding CRP to the MELD score increases its predictive value (Table 7 ).

The data testing what would happen if a variable were excluded from the model are presented. For the chosen model (an ascending model), it is shown that adding CRP to the model increases the difference from the initial model $\left(\chi^{2}=6.3\right.$, $p=0.011$ for MELD, and $\chi^{2}=7.8, p=0.005$ for MELD and CRP).

When considering the cut-off values MELD $14.7, \mathrm{CRP}>0.62$ as an indicator of the event rate of the absence of decompensation, odds ratio for the absence of decompensation was 11.407 (Cl 3.5-36) when adding CRP to the model. So, 
these values represent protective factors from decompensation $(\mathrm{p}<0.0001)$ (Table 8).

The ROC curve shows a $87.2 \%$ sensitivity and $58.3 \%$ specificity $(A \cup C=0.74 \mathrm{Cl} 0.6-0.8)$ for the MELD+CRP model.

\section{DISCUSSIONS}

C -reactive protein is produced in the liver under the influence of IL-6, IL-1 $\beta$, TNF- $\alpha$ (11). It was thought that CRP was produced exclusively by hepatocytes, but there are studies that suggest other sites of production including coronary artery smooth-muscle cells, inflamed kidneys, human neurons, alveolar macrophages and adipose tissue (12).

C-reactive protein reflects the synthesis of IL-6, a marker of activation of a proinflammatory response in cirrhosis (13).

The idea that patients with liver cirrhosis have a poor synthesis of CRP was shaken by the observation that elevated levels of CRP were seen even in CHILD C class patients with or without bacterial infection (11).

A Japanese study detected a cut-off point of $1.3 \mathrm{mg} / \mathrm{dL}$ for CRP in predicting the mortality of liver cirrhosis patients. There were CHILD C patients admitted to hospital for variceal hemorrhage with or without infection (14).

The results of the present study have shown that CRP was an independent factor of the influence of cirrhosis patients' prognoses, regardless of the MELD score value.

Furthermore, CRP is associated in a direct proportional manner with the rehospitalization rate.

C-reactive protein offers an objective value, which can be reproduced. It is not only a cheap, well-known approach available to clinicians but also, and above all, one that would not change either the manner of specimen collection for a patient or the steps preceding a transplant.

It is true that the CRP can be influenced by infections which do not involve the liver; however, it was proven that, regardless of the inflammation cause in cirrhosis patients, it negatively modifies their evolution (10).

Furthermore, SIRS can be camouflaged in cirrhosis patients; thus, the number of leukocytes may appear falsely normal due to hypersplenism, tachycardia is usually camouflaged by the beta-blockers administered to cirrhosis patients for the prophylaxis of esophageal varices, and tachypnea and hypercapnia are present in hepatic encephalopathy as well (10).

The peripheral arterial vasodilation hypothesis has been most influential in the theory of cirrhosis and its complications. Splanchnic arterial vasodilation contributes to portal hypertension and is the basis for manifestations such as ascites and hepatorenal syndrome, but there have been revealed gaps in the original pathophysiological interpretation of these complications. The expansion of knowledge on the mechanisms regulating vascular tone, inflammation and the hostmicrobiota interaction require a larger approach to advanced cirrhosis explaining all its manifestations. Indeed, multiorgan dysfunction and failure is likely to result from a interplay where the systemic spread of bacterial products represents the primary event. Endothelial molecular mechanisms responsible for arterial vasodilation and the release of a storm of inflammatory cytokines, reactive oxygen and nitrogen species are triggered by the consequent activation of the host innate immune response. Thus, the picture of advanced cirrhosis could be seen as the result of an inflammatory syndrome in contradiction with a simple hemodynamic disturbance (4).

Microorganisms from the systemic circulation determine hepatic endothelial disfunction during infections, which leads to hyperproduction of vasoconstrictors and consecutively, an increase in portal hypertension, leading to a greater risk of variceal hemorrhage (13).

C-reactive protein was identified as an independent predicting factor for the prognosis of cirrhotic patients, irrespective of the presence of decompensation. In conclusion, inflammation plays an important role in the pathogenesis of cir- rhosis (16).

A therapeutic argument for the inflammation theory in liver cirrhosis was that, in a laboratory study, the administration of celecoxib reduced the carbon tetrachloride-induced liver fibrosis in mice (15).

A Thai study (16) attempted to improve the MELD score by adding CRP, with favorable results for a group of 71 patients. The study was validated on 818 patients at the Mayo Clinic, in Rochester. As limits, the authors admitted the fact that the study was carried out only on patients with cirrhosis secondary to alcohol abuse, 
and validation was carried out by means of a retrospective approach (16).

Another study, conducted in France (10), found that introducing a fifth element (CRP) in the MELD score improved the mortality prediction capacity. However, as opposed to the previously mentioned study and the current one, the French study did not use the CRP value, but a qualitative variable (if CRP level changes or not within 15 days). Although the results improve the score, they make it more complicated and impose two CRP measurements in practice.

While Chirapongsathorn et al's study (16) involved patients hospitalized for cirrhosis complications, Di Martino et al (10) included patients with compensated cirrhosis as well.

The present study found that, for a cut-off point of $0,62 \mathrm{mg} / \mathrm{dL}$, CRP below this value predicted smaller chances for decompensation.

In a study carried out on patients with decompensated B viral liver cirrhosis, C-reactive protein associated to albumin, an already known factor of prognosis for cirrhosis, was an important factor, adding more power in predicting evolution of cirrhosis patients. Prognosis was influenced by the ratio between CRP and albumin $(C A R)$ and the one between leukocytes and monocytes (LMR). It has been shown that CAR was even more useful than LMR, MELD, and CHILD in predicting mortality (17).
C-reactive protein may help to the early detection of infections in hospitalized cirrhosis patients or may reflect the subclinical inflammation associated to the translocation of bacteria (18).

The small sample size was an important limitation of this study.

\section{CONCLUSION}

-reactive protein represents a prognostic factor for cirrhosis evolution.

The value of CRP influences the decompensation and rehospitalization rates.

A CRP value below the threshold of $0.62 \mathrm{mg} / \mathrm{dL}$ indicates a smaller probability of future decompensation in liver cirrhosis patients.

Conflicts of interest: none declared.

Ethics approval: This study was carried out with the approval of the hospital's Ethics Committee.

Acknowledgments: Thanks to Professor Victor Stoica, Internal Medicine and Gastroenterology $M D, P h D$, from "Carol Davila" University of Medicine and Pharmacy, Bucharest, Romania, for his guiding, and Alexandra Diaconeasa, Internal Medicine MD in Bucharest, for her help regarding the statistical processing of results.

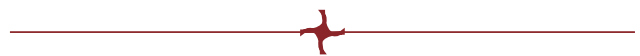

\section{R RFERENCES}

1. Di Martino V, Weil D, Cervoni J-P, Thevenot T. New prognostic markers in liver cirrhosis. World J Hepatol 2015;7:1244.

2. Ruf AE, Villamil FG. C-reactive protein and model for end-stage liver disease score: Have we found the fifth element? Liver Transplantation 2015;21:713-715.

3. Woodhouse CA, Patel VC, Goldenberg S, et al. PROFIT, a PROspective, randomised placebo controlled feasibility trial of Faecal Microbiota Transplantation in cirrhosis: study protocol for a single-blinded trial. BMJ Open 2019;9:e023518.

4. Bernardi M, Moreau R, Angeli P, et al. Mechanisms of decompensation and organ failure in cirrhosis: from peripheral arterial vasodilation to systemic inflammation hypothesis. Journal of Hepatology 2015;63:1272-1284.

5. Kamath PS, Kim WR. The model for end-stage liver disease (MELD). Hepatology 2007;45:797-805.

6. Peng Y, Qi X, Guo X. Child-Pugh versus MELD score for the assessment of prognosis in liver cirrhosis: a systematic review and meta-analysis of observational studies. Medicine 2016;95:e2877.

7. Burak KW, Meeberg GA, Myers RP, et al. Validation of the model of end-stage liver disease for liver transplant allocation in Alberta: implications for future directions in Canada.

Canadian Journal of Gastroenterology and Hepatology 2016;2016.

8. Cheng X-P, Zhao J, Chen Y, et al.
Comparison of the ability of the PDD-ICG clearance test, CTP, MELD, and MELD-Na to predict short-term and medium-term mortality in patients with decompensated hepatitis B cirrhosis. Eur J Gastroenterol Hepatol 2016;28:444.

9. Turco L, Garcia-Tsao G, Magnani I, et al. Cardiopulmonary hemodynamics and C-reactive protein as prognostic indicators in compensated and decompensated cirrhosis. J Hepatol 2018;68:949-958.

10. Di Martino V, Coutris C, Cervoni J, et al. Prognostic value of $\mathrm{C}$-reactive protein levels in patients with cirrhosis. Liver Transplantation 2015;21:753-760.

11. Lin K-H, Wang F-L, Wu M-S, et al. Serum procalcitonin and C-reactive protein levels as markers of bacterial 
infection in patients with liver cirrhosis: a systematic review and meta-analysis. Diagnostic Microbiology and Infectious Disease 2014;80:72-78.

12. Pieri G, Agarwal B, Burroughs AK. C-reactive protein and bacterial infection in cirrhosis.

Annals of Gastroenterology: Quarterly Publication of the Hellenic Society of Gastroenterology 2014;27:113.

13. Lee HH, Park JM, Han S, et al. A simplified prognostic model to predict mortality in patients with acute variceal bleeding.

Digestive and Liver Disease 2018;50:247-253.
14. Ichikawa $T$, Machida N, Kaneko H,et al. C-reactive Protein Can Predict Patients with Cirrhosis at a High Risk of Early Mortality after Acute Esophageal Variceal Bleeding.

Internal Medicine 2019;58:487-495.

15. Tang $S$, Huang $Z$, Jiang J, et al.

Celecoxib ameliorates liver cirrhosis via reducing inflammation and oxidative stress along spleen-liver axis in rats. Life Sciences 2021;272:119203.

16. Chirapongsathorn $S$, Bunraksa $W$, Chaiprasert A, et al. Adding C-reactive protein and procalcitonin to the model of end-stage liver disease score improves mortality prediction in patients with complications of cirrhosis.

J Gastroenterol Hepatol 2018;33:726-732.

17. Huang S-S, Xie D-M, Cai Y-J, et al.

$\mathrm{C}$-reactive protein-to-albumin ratio is a predictor of hepatitis

$B$ virus related decompensated cirrhosis: time-dependent receiver operating characteristics and decision curve analysis.

Eur J Gastroenterol Hepatol 2017;29:472-480.

18. Oikonomou T, Goulis I, Kiapidou S, et al. The significance of $\mathrm{C}$-reactive protein to albumin ratio in patients with decompensated cirrhosis.

Annals of Gastroenterology 2020;33:667. 